\title{
Genitopatellar syndrome
}

INSERM

\section{Source}

INSERM. (1999). Orphanet: an online rare disease and orphan drug data base.

Genitopatellar syndrome. ORPHA:85201

Genitopatellar syndrome is a rare congenital patellar anomaly syndrome characterized by patellar aplasia or hypoplasia associated with microcephaly, characteristic coarse facial features (microcephaly, bitemporal narrowing, large, broad nose with high nasal bridge, prominent cheeks and micro/retrog nathia or prognathism), arthrogryposis of the hips and knees, urogenital abnormalities and intellectual deficiency. 